\title{
Waiting pedestrians in the social force model
}

\author{
Fredrik Johansson, Anders Peterson and Andreas Tapani
}

\section{Linköping University Post Print}

N.B.: When citing this work, cite the original article.

Original Publication:

Fredrik Johansson, Anders Peterson and Andreas Tapani, Waiting pedestrians in the social force model, 2015, Physica A: Statistical Mechanics and its Applications, (419), 95-107.

http://dx.doi.org/10.1016/j.physa.2014.10.003

Copyright: Elsevier

http://www.elsevier.com/

Postprint available at: Linköping University Electronic Press

http://urn.kb.se/resolve?urn=urn:nbn:se:liu:diva-113715 


\title{
Waiting pedestrians in the social force model
}

\author{
Fredrik Johansson ${ }^{\mathrm{a}, \mathrm{b}, *}$, Anders Peterson ${ }^{\mathrm{b}}$, Andreas Tapani ${ }^{\mathrm{a}, \mathrm{b}}$ \\ ${ }^{a}$ Swedish National Road and Transport Research Institute (VTI) \\ SE-58195 Linköping, Sweden \\ ${ }^{b}$ Linköping University, Dept of Science and Technology (ITN) \\ SE-601 74 Norrköping, Sweden
}

\begin{abstract}
Microscopic simulation of pedestrian traffic is an important and increasingly popular method to evaluate the performance of existing or proposed infrastructure. The social force model is a common model in simulations, describing the dynamics of pedestrian crowds given the goals of the simulated pedestrians encoded as their preferred velocities.

The main focus of the literature has so far been how to choose the preferred velocities to produce realistic dynamic route choices for pedestrians moving through congested infrastructure. However, limited attention has been given the problem of choosing the preferred velocity to produce other behaviors, such as waiting, commonly occurring at, e.g., public transport interchange stations.

We hypothesize that: 1) the inclusion of waiting pedestrians in a simulated scenario will significantly affect the level of service for passing pedestrians, and 2) the details of the waiting model affect the predicted level of service, that is, it is important to choose an appropriate model of waiting.

We show that the treatment of waiting pedestrians have a significant impact on simulations of pedestrian traffic. We do this by introducing a series of extensions to the social force model to produce waiting behavior, and provide predictions of the model extensions that highlight their differences. We also present a sensitivity analysis and provide sufficient criteria for stability.
\end{abstract}

Keywords: social force model, waiting pedestrians, microscopic simulation

\footnotetext{
${ }^{*}$ Corresponding author, TEL: +46 13204036

Email addresses: fredrik.johansson@vti.se (Fredrik Johansson), anders.peterson@liu.se (Anders Peterson), andreas.tapani@vti.se (Andreas Tapani)
} 


\section{Introduction}

During the last couple of decades, the interest in simulation of pedestrian traffic has increased significantly. One reason for this is the recognition of walking as an important mode of transport, especially in connection to public transport, that should be prioritized to decrease the environmental impact of the transportation system. Another reason for the increased interest in pedestrian simulation is that development of accurate simulation tools have been made possible thanks to the increase in available computational power, and the advent of new models of pedestrian behavior. One of these models, the social force model (SFM) was introduced two decades ago by Helbing and Molnár [1], and has since then been frequently investigated in the literature, see e.g. [2-11], and is also implemented in commercial software [12]. It should be noted that the SFM is only one of a large class of force based models of pedestrian dynamics with a history ranging as far back as the work by [13].

The first applications of the SFM were mainly focused on simulating emergency evacuation of buildings, see for example Helbing et al. [14]. In such applications the goal of the pedestrians is to reach an exit as quickly as possible. However, the model has since its conception been extended to include a number of nontrivial behaviors, e.g. dynamic route choice [12] and pedestrians preferring to walk in a group [15]. Also, many variations of it have been proposed. Some examples to illustrate the diversity of the variations are: A. Johansson [16] experimented with the shape of the social field by letting it depend on the relative velocity of the interacting pedestrians, and performed a thorough comparison between the proposed and the traditional specifications of the field. Parisi et al. [17] extended the model to avoid that simulated pedestrians with a high preferred speed push other pedestrians forward or to the side when trying to walk by them. This was accomplished by temporarily setting the preferred speed to zero if the space immediately in front of the pedestrian is occupied. Lakoba et al. [18] introduced a numerically stable and efficient method to impose a minimum physical radius of the pedestrians, and also proposed an extension to include memory effects.

One nontrivial behavior that has received little attention, is that of waiting. Waiting pedestrians is a common feature in almost any normal traffic situation, specially at public transport interchange stations where pedestrians wait in front of information signs, in waiting rooms and at platforms. Accurate modeling of waiting pedestrians is important when 
using simulation to determine the level of service at a pedestrian facility, since groups of waiting pedestrians may reduce the capacity and create bottlenecks for passing pedestrians as showed by Davidich et al. [19]. In their work a spatially discrete model in which a waiting pedestrian is fixed in one cell and do not react to other pedestrians in its neighborhood is applied. The modeling of waiting pedestrians therefore becomes restricted to the choice of waiting position. In contrast, we will in this paper investigate a number of possible ways to include, in a spatially continuous model, the reaction of the waiting pedestrians to their surroundings.

Helbing and Molnár [1, 20] and Helbing et al. [21] mention, without further analysis, that group formation among pedestrians due to social bonds, and attraction to special places, such as street artists, can be modeled by attractive, often time dependent, forces similar to the repulsive forces used to model the aversion to walking close to walls, but with opposite sign. Such model would, however, as noted by Helbing and Molnár [20], result in behavior similar to that observed at rock concerts, with people struggling to get as close to the stage as possible; a behavior significantly differing from what is usually meant by waiting.

However, by modeling the operational behavior of the pedestrians using the SFM, it is possible to qualitatively reproduce a well known emergent phenomenon: the river-like streams of moving pedestrians through a standing crowd reported by Helbing et al. [21]. The ability of a model to correctly reproduce the formation of such streams may be important for the accuracy of predictions of macroscopic quantities, such as flow, since the formation of streams may facilitate the passage through a standing crowd in a similar way as dynamical lane formation facilitates bi-directional flow. Unfortunately dynamic lane formation and their correspondence in a standing crowd are hard to quantify.

In this article, we restrict the meaning of waiting to denote only the desire to stay at a certain place. This is the type of behavior typically displayed by people waiting at a railway platform, or in front of an information sign in a public transport interchange station. We do not include for example random strolling, or any such activity in the term waiting. Moreover, we assume that there exists a model determining if and where the simulated pedestrian prefers to stand, and investigate how this desire can be coupled to the SFM, thereby providing waiting agents with an operative behavior.

The purpose of this paper is to investigate the importance of accurate modeling of waiting pedestrians. More specifically, we hypothesize that: 1) the inclusion of waiting pedestrians in a simulated scenario will significantly 
affect the level of service for passing pedestrians, and 2) the details of the waiting model affect the predicted level of service, that is, it is important to choose an appropriate model of waiting.

The modeling of waiting pedestrians has two aspects, the reaction of passing pedestrians to the waiting, and the reaction of the waiting pedestrians to the passing. The main focus of the paper is on the first aspect at an aggregated level. However, the two aspects are closely connected and to understand the influence of the waiting pedestrians on the passing we also describe the reactions of the waiting pedestrians on an aggregated level. To investigate the effects of including waiting pedestrians and the sensitivity to the model used, a sequence of extensions to the SFM to include waiting pedestrians is presented, having simplicity as the guiding modeling principle.

We investigate the properties of the proposed model extensions and characterize the differences between the extensions by comparing their predictions. Furthermore we present a sensitivity analysis implying the sensitivity to the introduced model parameter and give sufficient criteria for the stability of the models. Finally, we conclude that the treatment of waiting pedestrians has significant impact on the predictions of pedestrian simulation models.

We start off in section 2 by describing the general type of modeling framework for which the proposed model extensions are intended, followed in section 3 by the presentation of the model extensions. In section 4 , the simulation scenario and the results of the simulations are presented, followed, in section 5 , by a discussion of the results, the limitations and the generalizability of the proposed model extensions, and possible further research directions.

\section{Modeling framework}

Since the waiting models presented in this paper are extensions to the SFM, we here give a brief review of the SFM, with focus on the features that are important for the development of the waiting models. A simulation framework based on the SFM can be viewed as a three layer model, with the levels corresponding to different mental processes of the pedestrians, ranging from instinctive behavior at the lowest level, up to premeditated behavior at the highest level, as illustrated in Figure 1. This categorization into three levels is based on the one presented in Hoogendoorn and Bovy [22].

To clarify and keep explanations brief, we will in the remainder of this article call a simulated pedestrian an agent, and allow ourselves to refer to an agent as it. 
The objectives of the agent are determined by the activity planning model at the strategical level: what should be done, where, and when. The result of the model at the strategical level is an origin-destination (O-D) matrix which is input to the tactical level. The route choice of the agent is determined by the route choice model at the tactical level, it gives the route that should be taken by the agent, given the O-D matrix supplied by the strategical level. The instinctive evasive maneuvers that are needed to avoid collisions between the agents on their way along the given routes are determined by the model at the operational level.



Figure 1: Structure of the simulation framework. The input data is an O-D matrix, which is used by the shortest path algorithm at the tactical level. The output of the tactical level, together with the individual preferred speeds of the agents provides the input $\mathbf{v}^{p}(\mathbf{x})$, the preferred velocity at any given point, $\mathbf{x}$, to the social force model at the operational level.

The work presented here is concerned with the modeling of waiting pedestrians at the tactical and operational level, that is, we do not consider the process of choosing a waiting area and waiting time. Thus, we do not model the strategical level, but regard the O-D matrix and activity plans of the agents as input data to the model. Further, at the tactical level, we let agents desire to take the shortest path, in space, between their respective origin and destination, assuming there are no other agents interfering. The instinctive behavior at the operational level is determined by the SFM which requires the preferred velocity of each agent at every moment in time as input. This preferred velocity is what connects the SFM to the tactical level; the shortest path model at the tactical level supplies the SFM with a 
preferred velocity such that if the agent moves with the preferred velocity at every time step it will follow the shortest path from its current position to its destination.

The SFM postulates that the behavior of pedestrians can be modeled by a set of forces that determine the accelerations of the agents. One of these forces, the preferred force, $F^{p}$, manifests the desire of an agent to move with its preferred velocity,

$$
\mathbf{F}^{p}=\frac{1}{\tau}\left(\mathbf{v}^{p}-\dot{\mathbf{x}}\right)
$$

where $\tau$ is a model parameter, $\mathbf{v}^{p}$ is the preferred velocity, and $\dot{\mathbf{x}}$ is the current velocity of the agent.

The agents avoid walking too close to each other due to velocity dependent anisotropic social forces. For discussion on different versions of the SFM see A. Johansson [16]. Details of the particular implementation of the SFM used to perform the simulations presented in this paper can be found in F. Johansson [23].

\section{Waiting models}

Waiting is a pedestrian behavior that includes all the three behavioral levels discussed in the previous section. The choice to wait or not to wait and in what area to wait, are choices belonging to the strategical level. We do not consider the strategical level here, but regard the results of these choices as input data. On the tactical level, waiting consists of choosing 1) where to wait within the chosen area, and 2) orientation, i.e. where to look. In addition, when the SFM is used, a preferred velocity must also be set. Finally, waiting also includes behavior on the operational level; how to react to other pedestrians while waiting.

The capabilities of the SFM have been demonstrated in many situations, such as reproducing the known real world phenomena dynamic lane formation, stripe formation and bottleneck oscillations. Furthermore, the properties of the model are well known, so it is preferable to make a minimum of modifications to it to model the operational level of the waiting behavior. The task of constructing a minimal waiting model extension then becomes that of choosing the preferred velocity of the agents while they are waiting.

Since the behavior of a waiting agent will be determined by a model differing from that of a passing agent, a transition must occur from the walking model to the waiting model. Thus we introduce two activities, 
walking and waiting, to specify if an agent behaves according to the standard model, or the waiting model.

We here propose a series of three waiting model extensions, starting from the most straightforward, followed by two more complex extensions to remedy the limitations of the preceding extension. In section 3.1 we discuss the general properties of the proposed waiting models, followed in sections 3.2 to 3.4 by a detailed description of each of the alternative extensions.

\subsection{Preliminaries}

There are many situations where pedestrians are waiting, and many of them have some feature that is special for that particular situation. Here we consider a situation common at public transport interchange stations. An object of interest, for example an information sign, is placed at position $\mathbf{x}^{f} \in \mathbb{R}^{2}$. We call $\mathbf{x}^{f}$ a focus point to emphasize that it can be any point that the agents are looking at. Associated with the focus point is a waiting area, $\Omega \subset \mathbb{R}^{2}$, from which the focus point is interesting to look at. The waiting area is a destination for a subset of the agents; before arriving to $\Omega$ their preferred velocity is directed along the shortest path from their current position to the closest point in $\Omega$. As an agent enters $\Omega$ it is assigned a waiting time and its activity changes to waiting for the duration of that time. This can be seen as a simple case of a finite state machine, which can be extended further with additional states, see for example Curtis et al. [24].

Usually in SFM based models, the orientation of an agent is taken to coincide with the direction of the preferred velocity, or occasionally, the actual velocity. However, a waiting agent may have both a vanishing speed and a vanishing preferred speed, which would make the traditional convention problematic. We let $\mathbf{e}=\mathbf{v}^{p} / v^{p}$, be the looking direction while walking, and

$$
\mathbf{e}^{w}=\frac{\mathbf{x}^{f}-\mathbf{x}}{\left|\mathbf{x}^{f}-\mathbf{x}\right|}
$$

be the looking direction while waiting, which is the only modification to the SFM we make, and it is unavoidable since the looking direction otherwise would become undefined for a stationary waiting agent. The waiting behavior is, with the above mentioned exception, achieved by modifying only the preferred velocity, at the tactical level of the model.

To set the notation and to clarify the relation between the dynamic entities of the models proposed in this paper the general geometry of the waiting scenario is depicted in Figure 2, followed by a similar figure for each waiting model presented. 


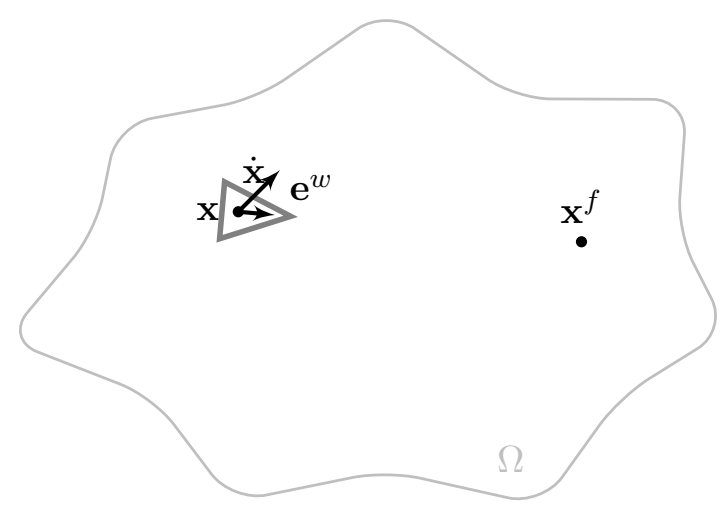

Figure 2: The geometry of the general waiting scenario. The looking direction while waiting, $\mathbf{e}^{w}$, is directed toward the focus point $\mathbf{x}^{f}$. For clarity the looking direction will be suppressed in the following figures and only indicated by the orientation of the triangle representing the agent. In this figure and the following figures, the velocity of the agent, $\dot{\mathbf{x}}$, is arbitrarily chosen.

\subsection{The Preferred Velocity (PV) model}

The simplest possible way to model a waiting pedestrian is to set the preferred velocity while waiting, $\mathbf{v}^{p w}$, to zero. We call this model the Preferred Velocity $(\mathrm{PV})$ model, and it is thus specified through $\mathbf{v}^{p w}=\mathbf{0}$, and $\mathbf{e}$ according to Equation (2). Thus the preferred force, Equation (1), of a waiting agent is reduced to $\mathbf{F}^{p w}=-\frac{\dot{\mathbf{x}}}{\tau}$. The geometry of the model is depicted in Figure 3 .

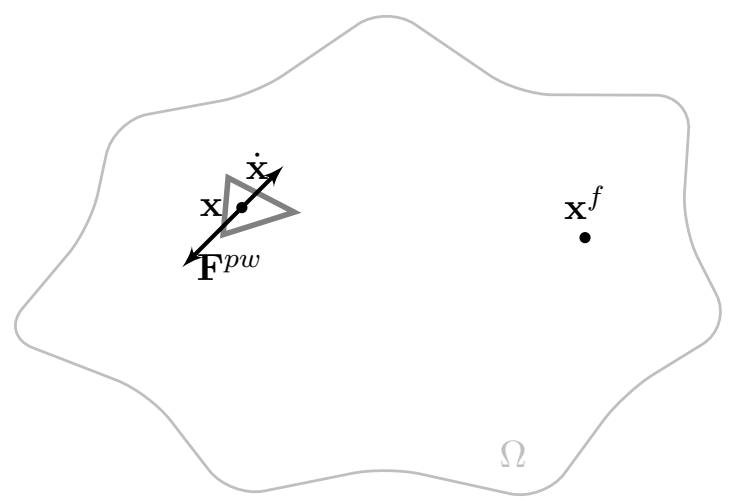

Figure 3: The geometry of the PV waiting model. The notation is as in Figure 2, with the addition of $\mathbf{F}^{p w}$, which in the preferred velocity model is directed in the opposite direction of the velocity $\dot{\mathbf{x}}$. 
This results in the equation of motion of a free waiting agent, i.e. an agent not affected by any other forces than its preferred force,

$$
\ddot{\mathbf{x}}=-\frac{\dot{\mathbf{x}}}{\tau} \text {. }
$$

As an agent enters $\Omega$ its activity switches to waiting, the preferred speed is set to zero, and the looking direction is set toward the focus point. The agent slows down exponentially with the time scale $\tau$.

A quick analysis of the model described above exposes an important feature of the model. Due to the SFM, the waiting agents are helpful; even a very sparse flow of passing agents will cause the waiting agents to drift away and end up along the boundaries of the walkable area. This behavior may of course be observed when all positions in the waiting area are equivalent, but not always, so it may be appropriate to, in some situations, include a desire of the agents to stay where they are.

\subsection{The Preferred Position (PP) model}

As mentioned above, in some situations it is unreasonable for a waiting pedestrian to be as ignorant of its waiting position as the agents in the PV model. This can be dealt with by assuming that a waiting agent has a preferred waiting position, $\mathbf{x}^{p w}$, to which it returns after being perturbed by a passing agent. We hence call the resulting model the Preferred Position (PP) model.

One such situation is when a queuing is modeled. Then, during the time the queue does not move, each pedestrian in the queue will have a very specific preferred position, but will be prepared to temporarily deviate from it in order to let a non queuing pedestrian pass through the queue. Another example is commuters waiting on a railway platform. Then it is not uncommon that they know where the train doors will appear, and thus position themselves accordingly.

The choice of $\mathbf{x}^{p w}$ belongs at a higher level in the model hierarchy, so here we assume that each agent has been assigned a preferred waiting position, and discuss the details of such a model in section 4 .

To make the agents return to their preferred position, the preferred waiting velocity, $\mathbf{v}^{p w}$, is set to be directed toward the preferred position in the PP model. Further we assume that the preferred speed continuously decreases as the distance to $\mathbf{x}^{p w}$ decreases, and that it vanishes if the agent is at its preferred position. Finally we assume that $\mathbf{v}^{p w}$ is equal to the agents regular preferred speed when it is far from $\mathbf{x}^{p w}$. 
We identify the simplest possible model obeying these assumptions as the one where the preferred speed increases linearly with the distance to the preferred position until it reaches the regular preferred speed $v^{p}$ at a distance $d$ from $\mathbf{x}^{p w}$. This can be seen as a linear approximation of the general model described in the previous paragraph. More advanced functions describing the decrease of the preferred velocity as the agent approaches the preferred position can be expected to result in behavior differing slightly from the behavior described by this linear approximation.

Thus we obtain the following specification of the preferred velocity while waiting,

$$
\mathbf{v}^{p w}(\mathbf{x})= \begin{cases}v^{p}\left(\mathbf{x}^{p w}-\mathbf{x}\right) / d, & \left|\mathbf{x}^{p w}-\mathbf{x}\right| \leq d, \\ v^{p}\left(\mathbf{x}^{p w}-\mathbf{x}\right) /\left|\mathbf{x}^{p w}-\mathbf{x}\right|, & \left|\mathbf{x}^{p w}-\mathbf{x}\right|>d\end{cases}
$$

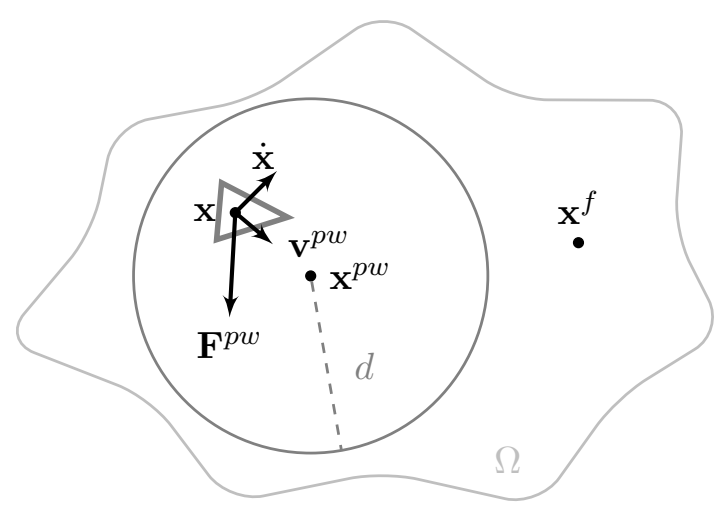

Figure 4: The geometry of the PP waiting model. The preferred position, $\mathbf{x}^{p w}$, is fixed and arbitrarily chosen in the figure. The preferred velocity, $\mathbf{v}^{p w}$, is due to Equation (3) always directed toward the preferred position. The distance $d$ is the radius of the disc within which $v^{p w}$ decreases linearly, from $v^{p w}=v^{p}$ at the border, to $v^{p w}=0$ at $\mathbf{x}^{p w}$.

The value of the model parameter $d$ can be chosen by considering the motion of a free agent in the proximity of its preferred position. Choose coordinates such that $\mathbf{x}^{p w}$ is at the origin (see Figure 4). Within the distance $d$ of $\mathbf{x}^{p w}$, the equations of motion become,

$$
\ddot{\mathbf{x}}+\frac{1}{\tau} \dot{\mathbf{x}}+\frac{v^{p}}{\tau d} \mathbf{x}=\mathbf{0},
$$

which in each dimension is the equation of a damped harmonic oscillator with damping coefficient $\zeta=\sqrt{d / 4 v^{p} \tau}$. We choose the value of $d$ by demanding 
that the agent slows down and stops at the preferred position as quickly as possible, without oscillating. This means that we demand that the harmonic oscillator, Equation (4), is critically damped $(\zeta=1)$, which implies that $d=4 v^{p} \tau$.

The PP waiting model produces the expected behavior: when perturbed by a passing agent, a waiting agent moves out of the way and then returns back to its preferred position as the perturbing agent has passed. Thus the potential problem with the drifting agents in the PV model is solved. However, another behavior is produced that may be unrealistic in some situations: if a stream of passing agents perturb the waiting agents, the waiting ones will persistently return to their preferred position as soon as a gap appears. In the two example applications mentioned in the beginning of this section, queuing and waiting where train doors are expected, this may indeed be a realistic behavior, but in some situations the preferred position may be uncomfortable due to lots of passing pedestrians, and a more pragmatic behavior of the agent would be more realistic. One way to achieve this is to let the agent adjust the preferred position if it seems unattainable for an extended period of time.

\subsection{The Adapting Preferred Position (APP) model}

For some applications the agents in the PP model perhaps too stubbornly return to their preferred position. This can be solved by including the preferred position as a dynamic entity in itself. If the agent is not at its preferred position the preferred position will be dragged toward the actual position of the agent, at the same time as the agent walks toward it. We call this model the Adaptive Preferred Position (APP) model.

Let the preferred position have a mass $M$, and the agent have a mass $m$, usually $m=1$. Let the preferred position be subjected to a force $-\mathbf{F}^{p w}$. Since the preferred position reasonably should have a tendency to slow down and be stationary, and to guarantee the stability of the system, we also include a friction force $-k \dot{\mathbf{x}}^{p w}$, where $k$, the viscous damping coefficient, is a model parameter. Thus the waiting behavior in the APP model is described by

$$
\begin{aligned}
\mathbf{F}^{p w} & =\frac{1}{\tau}\left(\mathbf{v}^{p w}(\mathbf{x})-\dot{\mathbf{x}}\right), \\
M \ddot{\mathbf{x}}^{p w} & =-\mathbf{F}^{p w}-k \dot{\mathbf{x}}^{p w},
\end{aligned}
$$

where $\mathbf{v}^{p w}$ is according to Equation (3)(see Figure 5). 


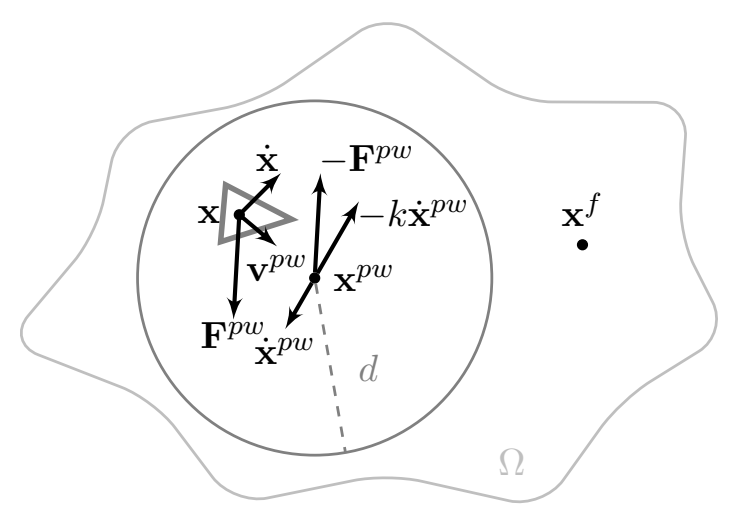

Figure 5: The geometry of the APP model. Notation as in previous figures with the addition of the variables determining the motion of the preferred position: the velocity of the preferred position, $\dot{\mathbf{x}}^{p w}$, arbitrarily chosen in the figure; the force dragging the preferred position toward the pedestrian, $-\mathbf{F}^{p w}$, and the friction force in the opposite direction of the velocity of the preferred position.

By choosing the model parameters

$$
k=\frac{M+m}{\tau},
$$

and,

$$
d=4 \tau v^{p} \frac{M+m}{M},
$$

as shown in Appendix A, a free agent will perform the same critically damped motion relative to the preferred position as in the PP model, and the speed of the preferred position of a free agent will decrease exponentially, thereby guaranteeing the stability of the model. By stability, we here imply that if the waiting agent is perturbed by an arbitrary force during a finite time, the agent and its preferred position will return to rest shortly after the perturbing force has stopped affecting the agent.

\section{Simulation results}

In this section, results are presented for a number of simulation experiments that were performed to investigate the properties of the proposed models. All the experiments are based on the same basic scenario, which is described in detail in section 4.1. In section 4.2 and section 4.3 comparisons of the 
results of running the basic scenario using the different proposed waiting models are given. In section 4.2 the effect of passing agents on waiting agents is examined through the comparison of the density distributions of waiting walkers across the corridor after the interactions have taken place. The purpose of this comparison is to quantify the difference in the reactions of the waiting agents in the three models. And then in section 4.3 the effect of waiting agents on the delay of passing agents is investigated through the delay compared to walking at the preferred speed that the passing agents suffer. The delay is a common way to evaluate the inefficiency of traffic flow, and in many circumstances the operator of the pedestrian infrastructure is interested in minimizing the delay. Therefore, delay is an important output of pedestrian traffic simulations, and its sensitivity to the model chosen to represent the waiting behavior should be investigated.

\subsection{The simulated scenario}

To investigate and compare the properties of the proposed waiting models a simple and general, but still realistic, simulation scenario was designed. The scenario to be modeled consists of a straight corridor, $50 \mathrm{~m}$ long and $8 \mathrm{~m}$ wide with an information sign, at $\mathbf{x}^{f}$, in the middle, in front of which a group of waiting pedestrians are gathered. Another group of pedestrians, with no interest in the information sign, passes by, interacting with the waiting pedestrians. The simulated scenario is depicted in Figure 6.

The passing agents are created in one end, uniformly distributed over the width of the corridor, with a mean rate of $2.6 \mathrm{~s}^{-1}$. Their destination is located at the opposite end of the corridor, and the agents always aim for the closest point of the destination.

The distance from the origin to the waiting area is chosen long enough for most of the initial interactions between the passing agents to disappear, but still short enough to prevent the group to disperse due to the differences in preferred speed among the agents.

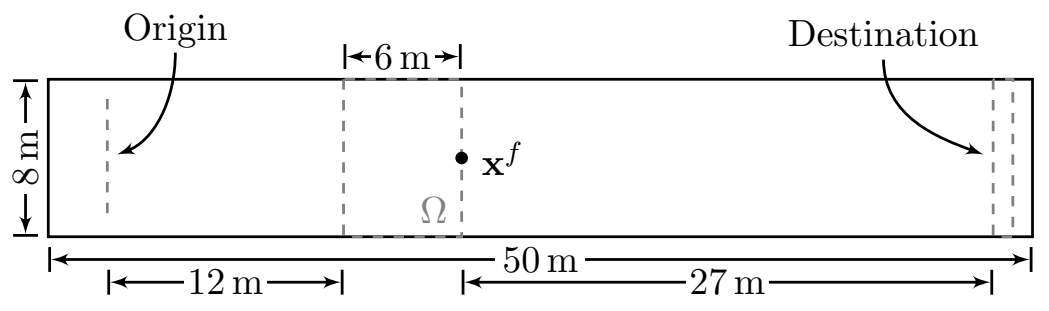

Figure 6: The geometry of the simulated scenario. 
Each simulation starts with no agents present. The waiting agents are created at the origin and moves toward the waiting area $\Omega$, which acts as a destination. When an agent with $\Omega$ as destination enters $\Omega$, the agent starts waiting, that is, its behavior becomes governed by one of the three waiting models described above. The passing agents are created $10 \mathrm{~s}$ after the waiting agents were created, also at the origin.

In the PV model the waiting agent just slows down while turning its field of view toward $\mathbf{x}^{f}$. In the PP and APP models, as the agent enters $\Omega$, it is assigned a preferred position, $\mathbf{x}^{p}$, on the line segment connecting $\mathbf{x}^{f}$ and the agent, such that $\left|\mathbf{x}^{p}-\mathbf{x}^{f}\right|=2 \mathrm{~m}$. The agent turns its field of view toward $\mathbf{x}^{f}$ and walks toward $\mathbf{x}^{p}$, while slowing down.

The three waiting models proposed in this paper differ in how the waiting agents behave when, but most importantly after, they are perturbed by other agents. Also the duration of the perturbation has different effects in the three models. To make a proper comparison of the models, the number of passing agents should be varied so that such effects can be accounted for. Two experiments were performed for each model, one with 10 passing agents and one with 100 passing agents. In both cases there were 10 waiting agents.

From observations of the animated output of the experiments it was clear that the models produce a behavior such that the passing agents tend to follow each other through the waiting group, in accordance with the expectations. However, due to the difficulties connected to quantifying this phenomenon, we do not try to directly compare it between the models.

\subsection{The density of waiting agents}

To quantify the effect of the passing agents on the waiting agents, the density distribution of waiting agents after the passing agents have passed is examined. To simplify the presentation we only study the distribution in the direction perpendicular to the flow, and integrate over the length of the corridor.

As a measure of the density we use the local agent density,

$$
\rho_{\sigma}(\mathbf{x}, t)=\sum_{i} \frac{1}{2 \pi \sigma^{2}} \exp \left(\frac{-\left(\mathbf{x}-\mathbf{x}_{i}(t)\right)^{2}}{2 \sigma^{2}}\right),
$$

where $\sigma$ is the scale of an agent in the continuous density field, $\mathbf{x}_{i}$ the location of agent $i$, and the sum is taken over all agents. Since the social force between agents has a range scale $\sigma_{\mathrm{SFM}} \approx 0.6 \mathrm{~m}$, see Zanlungo et al. [25] and A. Johansson [16], we use this as the scale of an agent, as proposed in 
F. Johansson [26]. This measure of the density has the advantage that it always results in a continuous density field, to the cost that it gives a small density where there cannot be any pedestrians.

The PV model is expected to result in a density distribution with peaks close to the walls of the corridor, since the waiting agents moving according to this model will not return to their original position after being perturbed by the passing agents. This effect should be more pronounced with more passing traffic. The PP model on the other hand is expected to result in a distribution with a peak in the middle of the corridor, since the agents moving according to this model always return to their preferred position. Finally, the APP model is expected to result in a distribution somewhere in between the PV and the PP model, and to be sensitive to the volume of the passing traffic.

The results of the experiments including 10 waiting and 10 passing agents are presented in Figure 7. The density distribution across the corridor is calculated by first calculating the local mean density field at a moment in time shortly after all the passing agents have passed. This field is then integrated over the length direction of the corridor to obtain a distribution for each repetition of the simulation. The mean of the distributions of all 100 replications are then calculated together with the standard error of the mean in each point.

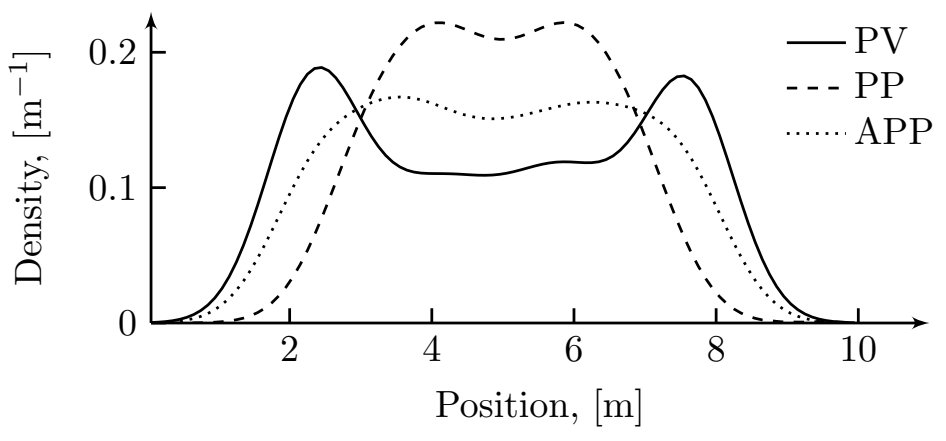

Figure 7: The mean density of the 10 waiting agents across the corridor after the 10 passing agents have passed by. The mean is taken in each point over 100 repetitions of the simulation. The standard error of the mean is equal to or less than $0.0042 \mathrm{~m}^{-1}$ in all points. The mass parameter in the APP model is chosen to $M=4$.

In Figure 8, the corresponding results of the experiments involving 100 passing agents are presented. 


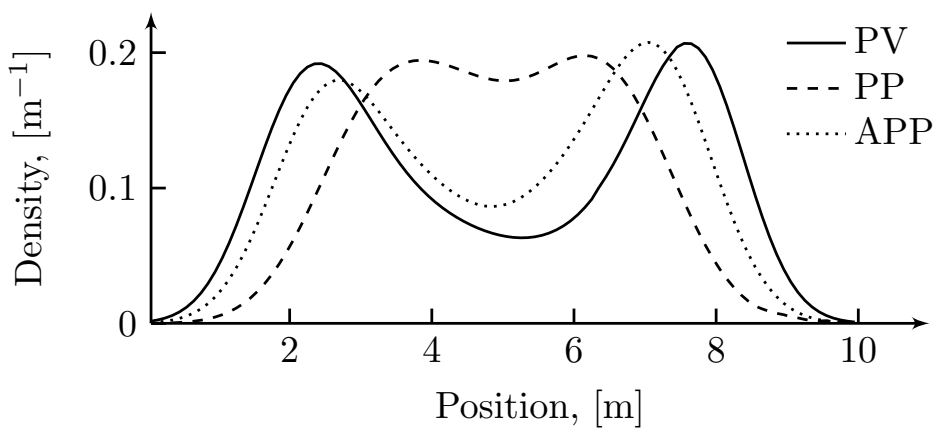

Figure 8: The mean density of the 10 waiting agents across the corridor after the 100 passing agents have passed by. The mean is taken in each point over 100 repetitions of the simulation. The standard error of the mean is equal to or less than $0.0102 \mathrm{~m}^{-1}$ in all points. The mass parameter in the APP model is chosen to $M=4$.

As can be seen in Figure 7 and Figure 8, the different models behave as expected. The distribution obtained from the experiments with the PV model exhibits clear peaks close to the corridor walls in both experiments. In the experiment with 10 passing agents there are some waiting agents that are located in the middle of the corridor after interacting with the passing agents, but in the experiment with 100 passing agents almost all waiting agents are pushed to the sides.

The PP model results in almost identical distributions regardless of the passing traffic volume. The waiting agents are concentrated to the middle of the corridor. Finally the APP model results in a slightly spread out centered peak in the experiment with 10 passing agents, while resulting in an almost identical distribution as the PV model in the experiment with 100 passing agents.

The results of the APP model presented above were obtained by setting the mass parameter to $M=4$, and as usual $m=1$. To investigate the sensitivity of the model to variations of the value of $M$, a series of experiments were performed where the value of $M$ was varied from 1 to 64 . It is not reasonable to let $M$ attain values below 1 , since this would imply a shorter time scale for the dynamics of the preferred position than for the agent itself. The hypothesis was that the result would vary from being similar, but not identical, to the PV model for low values of $M$, to becoming more and more similar to the PP model as $M$ increases. Note that the APP model, Equation (5), becomes identical to the PP model in the limit of infinite $M$. 
The results of simulations with four values of $M$ are presented in Figure 9 and Figure 10. As can be seen in Figure 9, the result is not overly sensitive to the value of $M$ : there is a fourfold increase in the value of $M$ and still the shift in the distribution is gradual. This implies that there are no sensitivity problems related to the choice of the value of $M$. This is important since the value must be obtained through calibration since it neither is directly observable, nor does it have a clear behavioral or physical interpretation that can facilitate an a priori determination of its value. As expected, the experiment with a high value of the parameter, $M=64$, gives a distribution almost identical to that from the experiment with the PP model in Figure 7, and the experiment with a low value, $M=1$, gives a distribution indicating that a significant shift toward the corridor walls has taken place. This behavior is expected since the mathematical formulation of the APP model is identical to the PP model in the limit of infinite $M$.

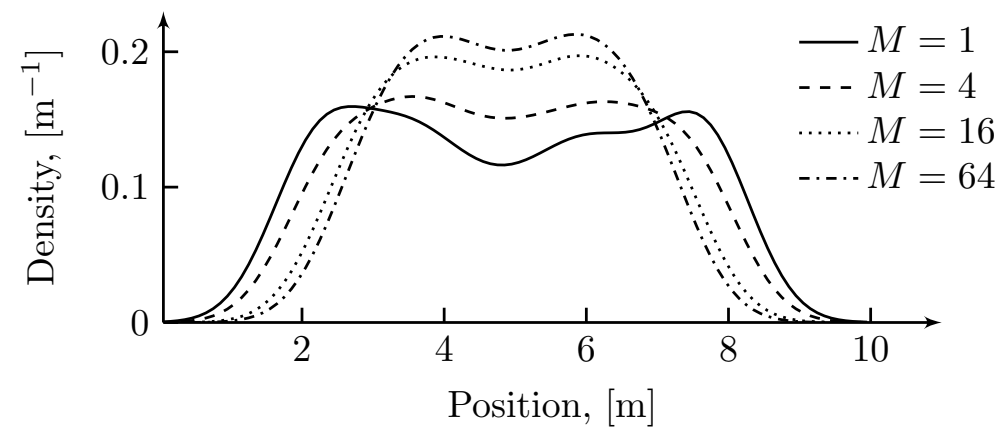

Figure 9: The mean density of the 10 waiting agents across the corridor after the 10 passing agents have passed by in the APP model experiments. The mean is taken in each point over 100 repetitions of the simulation. The standard error of the mean is equal to or less than $0.0040 \mathrm{~m}^{-1}$ in all points.

In Figure 10 we can see the transition between two different behaviors as the parameter value varies: for low values of $M$ the waiting agents are pushed to the sides, and stay there, while for large values they immediately return to the middle of the corridor. A similar difference can be seen when comparing the results of the APP model with $M=4$ in Figure 7 to the corresponding one in Figure 8.

\subsection{The delay of the passing agents}

To quantify the effect of the presence of waiting agents on the passing agents, the extent to which the passing agents are delayed is examined. This is 


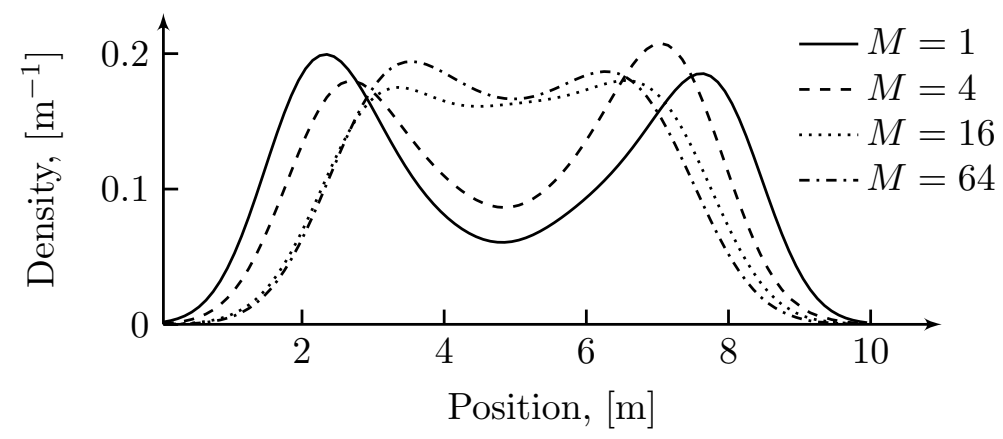

Figure 10: The mean density of the 10 waiting agents across the corridor after the 100 passing agents have passed by in the APP model experiments. The mean is taken in each point over 100 repetitions of the simulation. The standard error of the mean is equal to or less than $0.0105 \mathrm{~m}^{-1}$ in all points.

important since the delay, or walking time, is one of the most important outputs of a pedestrian simulation study, and if this quantity is sensitive to the way waiting pedestrians are modeled, then care must be taken when choosing waiting model in practical applications.

Since the waiting agents in the different models are more or less inclined to move out of the way it is expected that this will result in differences in the delay of the passing agents. The PV model is expected to predict the least delay, while the PP model is expected to produce the most.

The mean delay of the passing agents was calculated for each of the experiments and can be found in Figure 11. The delay of an agent is defined as the difference between its experienced travel time and the time it would have taken if it had walked along its shortest path at its preferred speed. As can be seen from the figure there are significant differences between the predicted delay of the different models. This becomes specially apparent if we consider only the additional delay caused by the waiting agents, that is the difference to the reference scenario " $\mathrm{N}=0$ " without waiting agents. Then we see that in the PP model the waiting agents causes an additional $30 \%$ and $34 \%$ delay compared to the PV model in the 10 and 100 passing agents experiments respectively.

The mean total delay is a highly aggregated measure of the delay inflicted on the passing agents. To investigate this in more detail it is possible to study the distribution of individual delays. It is expected that the PV model should produce a smaller frequency of agents with a high delay compared to 


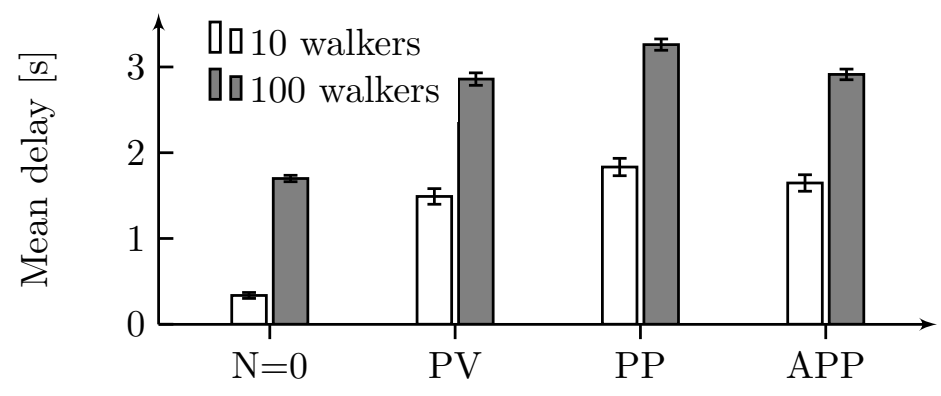

Figure 11: The mean delay of the passing agents in the experiment with 10 passing agents (white bars), and 100 passing agents (gray bars). In both cases there are 10 waiting agents. Also included is a reference scenario without any waiting agents ("N=0"). The errorbars represent the $95 \%$ confidence intervals of the estimated means, based on 100 repetitions of the simulation. In the simulations with the APP model the mass parameter was set to $M=4$.

the PP model, and also to some extent the APP model. Since high delays are expected to be connected to situations when a waiting agent is located almost exactly straight in front of an approaching agent, or when the passing agent has to perform several avoidance maneuvers, these situations should be less frequent when the waiting agents adjust their waiting positions.

The total delay of each passing agent from 100 repetitions of the simulation for each model was collected into a 50 bins histogram. The results can be seen in Figure 12. The figure confirms the expectations; the PV model produces fewer high delay results than the PP model.

\section{Discussion}

The comparison of the output of the different models clearly indicates that the choice of waiting model is of high importance for the applicability of pedestrian simulation.

If we compare the density distributions of waiting agents when the APP model is used in Figure 7 and in Figure 8, we see that the distribution of waiting agents is highly dependent on the passing flow. In the first case with 10 passing agents we see just a slight widening of the central peak, while when 100 agents pass almost all the waiting agents are forced to the sides. We see a similar difference when comparing the distributions from experiments with a fixed volume, and varying value of $M$, Figure 9 and 


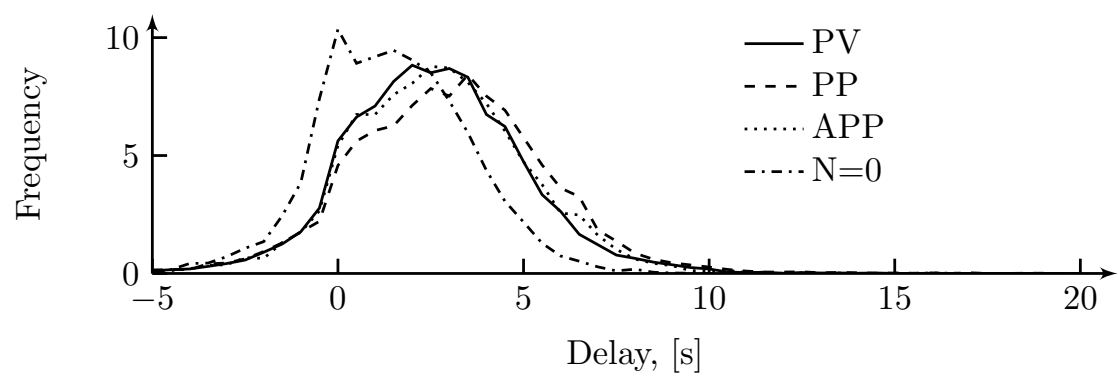

Figure 12: The distribution of individual total delays in the experiment with 100 passing agents and 10 waiting agents for the three different models and, for reference, the delay distribution when there are no waiting agents. In the distributions, agents from 100 repetitions of the simulation are included. The distributions are histograms with 50 bins between $-5 \mathrm{~s}$ and $20 \mathrm{~s}$.

Figure 10. These two observations indicate that it may be possible to find a suitable value of $M$ from field observations.

The simulated scenario, described in section 4.1, may at a first glance seem overly specific. However, it should be noted that the scenario could easily be generalized by allowing the focus point $\mathbf{x}^{f}$ to be chosen individually by each agent and even have it depend on time. From the perspective of the waiting models, the only assumptions we make are that there is a transition to the waiting activity, and an externally given looking direction, motivated by the environment.

Of course, the models may also be generalized by inclusion of additional parameters, or allowing for a more general form of the dependence of the preferred speed on the distance to the preferred position, but doing so would destroy the simplicity of the models and complicate the analysis of the model properties since the analogue with the well known physical system of the harmonic oscillator would be lost. However, the models proposed here will remain useful as first approximations.

There is one important limitation in the applicability of the models. They all assume that waiting pedestrians still react to surrounding pedestrians according to the SFM. This results in waiting agents that are quite sensitive to their surroundings, which in some situations not corresponds to reality, for example if the waiting pedestrians have lots of luggage. This limitation may be rectified by the introduction of an additional parameter that determines the sensitivity of waiting agents to forces from the surrounding agents by simply scaling down the waiting agents reaction to these forces by a factor, 
thus leading to more stationary waiting agents. However, using scaling of the reactions is not trivial since it disturbs the delicate balance between forces in the SFM, but is an interesting subject for future research.

Of course, the accuracy of the waiting models is limited by the accuracy and drawbacks of the SFM, and of force based models in general. However, the proposed waiting models do not exhibit any oscillatory behavior, and are by construction guaranteed not to do so. In addition to this, the waiting models themselves are not calibrated or validated using observational data. This is, however, not a problem in this study since the purpose is to estimate a lower bound on the effect of including waiting agents, and to estimate the sensitivity of the results with respect to the specifications of the waiting model. One should also note that there are many specifications of the SFM. However, the waiting models proposed here do not depend on the precise form of any such specification, but could be applied to many force based models. The detailed numerical results do, however, depend on the specific type of model, as well as the parameters used.

In this paper, the route choice of the passing pedestrians, that is their desired velocity, was assumed to be static. It has been shown that using a dynamic route choice model that to some extent directs the agents closer to a quickest path, rather than the static shortest path, may result in more realistic behavior [12]. Also, the study by Davidich et al. [19] indicate that passing pedestrians prefer to walk around a waiting group rather than through it, indicating that dynamic route choice is important. However since there seems to be no consensus in the literature on how such a dynamic route choice model should be constructed we chose to investigate the proposed waiting models in a scenario where it can be assumed that dynamic route choice will have a small effect on the results.

It is clear that a more accurate model governing the choice of the preferred position in the PP and APP models should take into consideration several factors that was omitted in this paper: the distribution of waiting agents already present, the passing flow or the waiting agents estimate of the future passing flow, and possibly also conditions affecting the attractiveness of the waiting area, such as the readability of a sign, availability of shelter, or the prospect of being able to board a public transport vehicle. There exists some reported observations on where people prefer to wait under specific circumstances, such as on railway platforms [27] and in railway stations [19], but these observations are on a higher level of aggregation. Investigating the effect of different models for the choice of the preferred waiting position is an important line of future research. 


\section{Conclusions}

We have proposed, and investigated the properties of, three models for simulation of waiting pedestrians in SFM based simulation frameworks. The models are simple and applicable as first approximations of waiting behavior, and due to their simplicity we were able to provide sufficient criteria for their stability.

Through a series of simulation experiments we showed that the differences between the predictions of the three proposed models are significant. We can conclude that the treatment of waiting pedestrians is important for the predictions of pedestrian simulation models. This implies that any application of SFM based pedestrian simulation to scenarios with a significant amount of waiting pedestrians is associated with uncertainties; further model development and calibration is needed to provide reliable results.

Further research inspired by the work presented includes the study of to what extent the reactions of pedestrians to surrounding traffic are suppressed when they are waiting, and how the choice of a preferred waiting position can be dynamically modeled in a general pedestrian traffic scenario.

\section{Acknowledgments}

The work presented here was made possible thanks to the generous support of the Swedish Transport Administration, TRV 2013/13329.

\section{Appendix A. Stability of the adapting preferred position model}

Here we show that choosing $k=(M+m) / \tau$, and $d=4 \tau v^{p} \frac{M+m}{M}$, is a sufficient criterion for stability and results in the desired critically damped motion of an agent relative to its preferred position.

Consider a free, i.e. not subjected to any external forces, waiting agent within the distance $d$ of its preferred position. Inserting Equation (3) into Equation (5a) gives the equations of motion for the agent and its preferred position,

$$
\begin{aligned}
\ddot{\mathbf{x}}= & \frac{1}{\tau}\left(\frac{v^{p}}{d}\left(\mathbf{x}^{p w}-\mathbf{x}\right)-\dot{\mathbf{x}}\right), \\
M \ddot{\mathbf{x}}^{p w} & =-m \ddot{\mathbf{x}}-k \dot{\mathbf{x}}^{p w} .
\end{aligned}
$$

We introduce the location of the agent relative to the preferred waiting position,

$$
\mathbf{z}=\mathbf{x}-\mathbf{x}^{p w}
$$


Using $\mathbf{z}$ and $\mathbf{x}^{p w}$ transforms Equation (A.1) to,

$$
\begin{aligned}
\ddot{\mathbf{x}}^{p w}+\frac{1}{\tau} \dot{\mathbf{x}}^{p w} & =-\ddot{\mathbf{z}}-\frac{1}{\tau} \dot{\mathbf{z}}-\frac{v^{p}}{\tau d} \mathbf{z}, \\
\ddot{\mathbf{x}}^{p w}+\frac{k}{M+m} \dot{\mathbf{x}}^{p w} & =-\frac{m}{(M+m)} \ddot{\mathbf{z}},
\end{aligned}
$$

and we can see that the equations of motion of the relative position of the agent decouples from that of the preferred position if we choose $k=$ $(M+m) / \tau$,

$$
\begin{aligned}
\ddot{\mathbf{z}}+\frac{M+m}{\tau M} \dot{\mathbf{z}}+\frac{(M+m) v^{p}}{\tau d M} \mathbf{z} & =\mathbf{0}, \\
\ddot{\mathbf{x}}^{p w}+\frac{1}{\tau} \dot{\mathbf{x}}^{p w} & =-\frac{m}{(M+m)} \ddot{\mathbf{z}} .
\end{aligned}
$$

Now Equation (A.2a) is a harmonic oscillator which, if we choose $d=$ $4 \tau v^{p} \frac{M+m}{M}$, is critically damped. The solution of Equation (A.2a) inserted in Equation (A.2b) gives the motion of the preferred position, which, as a result of the exponentially decreasing right hand side, also is exponentially decreasing.

Thus we can guarantee that, for any initial conditions such that the agent is within a distance $d$ of the preferred position, the agent returns to its preferred position without oscillations, and the preferred position is dragged toward the agent, also without oscillations. This is true for any value of $M \geq m$, where larger $M$ results in slower and smaller adjustments of the preferred position.

To further indicate the stability of the model, we give an upper bound on the maximum speed of the preferred position. When the distance to the preferred position is greater than $d$ the motion of the agent is determined by the standard SFM preferred force, but guided toward a moving target,

$$
\begin{aligned}
\ddot{\mathbf{x}} & =\frac{1}{\tau}\left(v^{p} \frac{\mathbf{x}^{p w}-\mathbf{x}}{\left|\mathbf{x}^{p w}-\mathbf{x}\right|}-\dot{\mathbf{x}}\right), \\
M \ddot{\mathbf{x}}^{p w} & =-m \ddot{\mathbf{x}}-k \dot{\mathbf{x}}^{p w} .
\end{aligned}
$$

Since the speed of the agent is bounded by a maximum speed $v^{\max }$, the maximum preferred force is $m\left(v^{p}+v^{\max }\right) / \tau$. Assuming, in line with finding an upper bound, that this force could be sustained for a longer period of time, the motion of the preferred position would be described by

$$
M \ddot{x}^{p w}+\frac{M+m}{\tau} \dot{x}^{p w}=-\frac{m}{\tau}\left(v^{p}+v^{\max }\right) .
$$


Since the preferred position is created at rest, and its motion is described by Equation (A.3) if it is affected by the maximum possible force, the maximal speed of the preferred position is

$$
\dot{x}_{\max }^{p w}=\frac{m\left(v^{p}+v^{\max }\right)}{M+m} .
$$

Thus both the speed of the agent and the speed of its preferred position is bounded, and both decrease exponentially in the absence of external forces.

\section{References}

[1] D. Helbing, P. Molnár, Social force model for pedestrian dynamics, Physical Review E: Statistical, nonlinear and soft matter physics 51 (1995) 4282-4286. arXiv: cond-mat/9805244. doi:10.1103/PhysRevE. 51.4282 .

[2] G. Frank, C. Dorso, Room evacuation in the presence of an obstacle, Physica A: Statistical Mechanics and its Applications 390 (2011) 21352145. doi:10.1016/j . physa.2011.01.015.

[3] V. Ha, G. Lykotrafitis, Agent-based modeling of a multi-room multifloor building emergency evacuation, Physica A: Statistical Mechanics and its Applications 391 (2012) 2740-2751. doi:10.1016/j .physa.2011. 12.034.

[4] D. Helbing, I. J. Farkas, P. Molnár, T. Vicsek, Simulation of pedestrian crowds in normal and evacuation situations, Pedestrian and evacuation dynamics 21 (2002) 21-58.

[5] D. Helbing, L. Buzna, A. Johansson, T. Werner, Self-organized pedestrian crowd dynamics: Experiments, simulations, and design solutions, Transportation Science 39 (2005) 1-24. doi:10.1287/trsc.1040.0108.

[6] A. Johansson, D. Helbing, P. K. Shukla, Specification of the social force pedestrian model by evolutionary adjustment to video tracking data, Advances in Complex Systems 10 (2007) 271-288. arXiv:0810.4587. doi:10.1142/S0219525907001355.

[7] P. Ma, B. Wang, The escape of pedestrians with view radius, Physica A: Statistical Mechanics and its Applications 392 (2013) 215-220. doi:10. 1016/j.physa.2012.07.053. 
[8] D. Parisi, C. Dorso, Microscopic dynamics of pedestrian evacuation, Physica A: Statistical Mechanics and its Applications 354 (2005) 606-618. doi:10.1016/j.physa.2005.02.040.

[9] P. Saboia, S. Goldenstein, Crowd simulation: applying mobile grids to the social force model, The Visual Computer 28 (2012) 1039-1048. doi:10.1007/s00371-012-0731-y.

[10] A. Seyfried, B. Steffen, T. Lippert, Basics of modelling the pedestrian flow, Physica A: Statistical Mechanics and its Applications 368 (2006) 232-238. arXiv:physics/0506189. doi:10.1016/j . physa. 2005. 11.052.

[11] L. Wang, Q. Zhang, Y. Cai, J. Zhang, Q. Ma, Simulation study of pedestrian flow in a station hall during the spring festival travel rush, Physica A: Statistical Mechanics and its Applications 392 (2013) 24702478. doi:10.1016/j.physa.2013.01.044.

[12] T. Kretz, A. Große, S. Hengst, L. Kautzsch, A. Pohlmann, P. Vortisch, Quickest paths in simulations of pedestrians, Advances in Complex Systems 14 (2011) 733-759. arXiv:1107.2004. doi:10.1142/ S0219525911003281.

[13] K. Hirai, K. Tarui, A simulation of the behavior of a crowd in panic, in: Proceedings of the 1975 International Conference on Cybernetics and Society, 1975, pp. 409-411.

[14] D. Helbing, I. Farkas, T. Vicsek, Simulating dynamical features of escape panic, Nature 407 (2000) 487-490. arXiv: cond-mat/0009448v1. doi:10.1038/35035023.

[15] M. Moussaïd, N. Perozo, S. Garnier, D. Helbing, G. Theraulaz, The walking behaviour of pedestrian social groups and its impact on crowd dynamics, PLoS ONE 5 (2010) e10047. arXiv:1003.3894. doi:10.1371/ journal.pone. 0010047.

[16] A. Johansson, Data-Driven Modeling of Pedestrian Crowds, Ph.D. thesis, Technische Universität Dresden, 2009. URL: http://nbn-resolving. de/urn:nbn:de:bsz:14-qucosa-20900.

[17] D. Parisi, M. Gilman, H. Moldovan, A modification of the social force model can reproduce experimental data of pedestrian flows in normal 
conditions, Physica A: Statistical Mechanics and its Applications 388 (2009) 3600-3608. doi:10.1016/j .physa.2009.05.027.

[18] T. Lakoba, D. Kaup, N. Finkelstein, Modifications of the helbing-molnarfarkas-vicsek social force model for pedestrian evolution, SIMULATION: Transactions of the Society for Modeling and Simulation International 81 (2005) 339-352. doi:10.1177/0037549705052772.

[19] M. Davidich, F. Geiss, H. G. Mayer, A. Pfaffinger, C. Royer, Waiting zones for realistic modelling of pedestrian dynamics: A case study using two major german railway stations as examples, Transportation Research Part C: Emerging Technologies 37 (2013) 210 - 222. doi:10. $1016 / j \cdot \operatorname{trc} .2013 .02 .016$.

[20] D. Helbing, P. Molnár, Self-organization phenomena in pedestrian crowds, in: F. Schweitzer (Ed.), Self-Organization of Complex Structures. From Individual to Collective Dynamics, Gordon and Breach, London, 1997, pp. 569-577. arXiv: cond-mat/9806152.

[21] D. Helbing, P. Molnár, I. Farkas, K. Bolay, Self-organizing pedestrian movement, Environment and Planning B: Planning and Design 28 (2001) 361-383. doi:10.1068/b2697.

[22] S. Hoogendoorn, P. Bovy, Pedestrian route-choice and activity scheduling theory and models, Transportation Research Part B: Methodological 38 (2004) 169-190. doi:10.1016/S0191-2615(03)00007-9.

[23] F. Johansson, Pedestrian traffic simulation platform, Swedish National Road and Transport Research Institute, Linköping, 2013. URL: http://www.vti.se/en/publications/pdf/ pedestrian-traffic-simulation-platform.pdf.

[24] S. Curtis, S. Guy, B. Zafar, D. Manocha, Virtual tawaf: A case study in simulating the behavior of dense, heterogeneous crowds, in: Computer Vision Workshops (ICCV Workshops), 2011 IEEE International Conference on, 2011, pp. 128-135. doi:10.1109/ICCVW.2011.6130234.

[25] F. Zanlungo, T. Ikeda, T. Kanda, Social force model with explicit collision prediction, EPL (Europhysics Letters) 93 (2011) 68005. doi:10. 1209/0295-5075/93/68005. 
[26] F. Johansson, A. Peterson, A. Tapani, Performance evaluation of railway platform design using microscopic simulation, in: Proceedings of CASPT12, Santiago, Chile, 2012.

[27] W. H. K. Lam, C. Y. Cheung, C. F. Lam, A study of crowding effects at the hong kong light rail transit stations, Transportation Research Part A: Policy and Practice 33 (1999) 401-415. doi:10.1016/S0965-8564(98) 00050-0. 